\title{
Conspiracy Determines World War III with Reference to Several Windows' Channel in Connection with Earth Observation Satellite
}

\author{
Swapan Kumar Ghosh \\ Department of Mathematics, Narajole Raj College, Midnapore, India \\ Email: g_swapan2002@yahoo.com
}

How to cite this paper: Ghosh, S.K. (2018) Conspiracy Determines World War III with Reference to Several Windows Channel in Connection with Earth Observation Satellite. World Journal of Mechanics, 8, 107-111.

https://doi.org/10.4236/wjm.2018.84009

Received: March 23, 2018

Accepted: April 27, 2018

Published: April 30, 2018

Copyright $\odot 2018$ by author and Scientific Research Publishing Inc. This work is licensed under the Creative Commons Attribution International License (CC BY 4.0).

http://creativecommons.org/licenses/by/4.0/

\section{(c) (i) Open Access}

\begin{abstract}
Thermonuclear reaction exerts its influence of X-emission to produce several windows' channel in the presence of an oscillator under electrical relay circuit with a decisive importance to a radiofrequency Earth observation satellite. Indian Television Network (National Channel) has introduced a radiofrequency accelerator to produce $\mathrm{X}$-emission at resonance with an activation of artificial human environment under relay analogy in the presence of an Earth observation satellite. Thermonuclear reaction communicates several windows' channel via Earth observation satellite. Star Television network communicates an artificial human environment under the influence of a relay circuit with different pulse code units of human brain with an active influence of an artificial sensation to generate the loss of humanity around the world.
\end{abstract}

\section{Keywords}

Thermonuclear Reaction, Radio Emission, X-Emission, Earth Observation Satellite, Window Channel

\section{Introduction}

As referred to a controlled thermonuclear fusion reaction of the Sun with reference to author investigation of Ghosh [1], it is a decisive importance to a radiofrequency accelerator to produce radio emission in a vacuum. In relevance to a controlled thermonuclear fusion reaction of the Sun, plasma fusion interacts with the supercritical state in the presence of a strong magnetic field by means of a magnetic mirror with the Sun. In compliance with the study of Ghosh [2] [3] Ghosh et al. [4] and Ghosh [5] it is stated that Star Television (United Kingdom) 
network exerts its influence of several windows' channel in a vacuum leading to an artificial human environment around the world in the presence of a radiofrequency accelerator. It is important to note that in the year of 1962, Star Television (United Kingdom) has introduced a radiofrequency satellite with the appliance of underground $\mathrm{X}$-emission at resonance in a vacuum to activate species all over the world. The simultaneous action of Russian Earth observation satellite and United Kingdom Earth observation satellite leads to a vacuum to expedite radiofrequency voltage to produce X-emission into Star Television network through radiofrequency driver so that Star Television network has a great deal of advantages to produce $\mathrm{X}$-emission to increase radiofrequency voltage and the synchronization of ultraviolet radiation and $\mathrm{X}$-emission of shorter wave length become a X-ray burst in a vacuum. It is noticed that a relay circuit is introduced to produce artificial sensation via several windows' channel to work with species throughout the world. In the year of 1970, it has been found that Indian Television network (National Channel) has introduced radiofrequency accelerator in co-operation with Star Television network to produce X-emission through radio frequency driver to create artificial sensation and that becomes a disaster of humanity. The configuration of the Sun in the presence of a radiofrequency accelerator leads to a vacuum so that frictional layer at the boundary suddenly sets into motion. It reveals that in space, pressure is far below normal atmospheric pressure and the flow is oscillating in nature with reference to a driving force. Since the Sun lies in a vacuum, the Earth is put into a vacuum. Indeed, radio emission deals with neurocybernetics in compliance with an electrical relay circuit to create artificial human sensation with a different pulse code unit of human brain. Nevertheless, a destructive influence of the society via radiofrequency satellite in association with Indian Television network (National Channel) is subjected to artificial motion picture with several windows' channel to exhibit species in different parts of the world. It is worthy to note that United Kingdom Earth Observation Satellite to put into hold on Russian Earth observation satellite with the inclusion of American Star War programme is the communication of several murders via Star Television network in association with Indian Television network. X-emission is a powerful evidence of Air Crash. The black hand Mr. Bikash Sinha and his alliance, Saha Institute of Nuclear Physics, Kolkata (West Bengal, INDIA) were recognized in connection with the murder of a Russian President, Dr. Anthrapov (D.Sc in Physics). It is noticed that the other black hand was sent to Russian Space Laboratory under the supervision of Russian Ladies organizing committee who has been involved with several murder around the world via Indian Television Network (Kolkata, West Bengal, INDIA). It is important to note that in the year of 1978, Russian Ladies Organizing Committee has introduced radio emission in the presence of radiofrequency accelerator into Indian Television network (National Channel, Kolkata, West Bengal, India) to activate artificial human environment via several windows' channel with reference to neurocybernetics. In this situation, $\mathrm{X}$-emission is produced to increase 
radiofrequency voltage. Every transmission operations were occupied by a radiofrequency voltage to become a significant effect of X-ray burst into electrical transmission operation. Mr. Bikash Sinha (International criminal) was arrested by the Central Bureau of Investigation Special Branch, New Delhi, INDIA in connection with the murder of Mrs. Indira Gandhi, Late Prime Minister of India when he was the Director, Saha Institute of Nuclear Physics, Kolkata, West Bengal, INDIA. Before that incident, it has been shown that a secret sexual relation was developed between Mr. Bikash Sinha and one of the queen of England via radiofrequency satellite, United Kingdom and a marriage proposal was given by the queen of England in order to release Mr. Bikash Sinha in connection with the murder of Late Prime Minister of India, Mrs. Indira Gandhi. Mr. Bikash Sinha was released by the Government of India. Now, Mr. Bikash Sinha is a citizen of United Kingdom and Switzerland. It is important to note that a secret issue of Bofors Gun deal with India came from Italy and Switzerland in connection with Mr. Bikash Sinha and his alliance subject to severe conspiracy. Star Television network deals with thermonuclear reaction in the presence of a magnetic mirror permeated by a radio frequency accelerator to produce underground X-emission with the help of a radiofrequency satellite and ionization takes place of species concentration subjected to a relay circuit via United Kingdom Earth observation satellite to show space accident with reference to $\mathrm{X}$-emission and radio emission lies in its behaviour of species to expedite artificial sensation. The transmission of radio wave with a decisive importance to radiofrequency voltage to deal with Indian Television circuit network in order to trap electrical transmission circuit such as electrical transmission, Telephone, radar communication via an electrical relay circuit box.

\section{Scientific Report}

The foundation of India Television Network (National Channel) exerts its influence of Russian Earth observation Satellite to expedite thermonuclear reaction in a vacuum. An oscillating current flow is generated in a vacuum in the presence of a radiofrequency satellite. In the presence of a radiofrequency accelerator with Indian Television network with the inclusion of Star Television network, radio emission determines the emission of radiofrequency electromagnetic radiation by oscillating current flow. A relay circuit was introduced in the presence of a magnetic mirror to deal with species via several windows' channel. Neurocybernetics deals with radio emission with the growing of species in the presence of magnetic mirror to activate human environment at different pulse code unit of human brain. In such a situation it reveals that the assassination of Mr. Rajib Gandhi (Late Prime Minister of India) deals with the neurocybernetics to put into him subject to an artificial human environment with different pulse code unit and the murder of Mr. Rajib Gandhi is an explanation of X-emission to increase radiofrequency voltage that becomes X-ray burst on his brain due to thermonuclear flashes. A radiofrequency satellite deals with radio emission to 
exert its influence of an artificial human sensation with a decisive importance to an electrical relay circuit. Human brain is an important feature of neuroscience by applying pulse oscillator under the system of Maxwell's equation in such a way that impulse under electrical oscillation is subjected to a kinetic energy of the system which undergoes a continuous process through neuron loops to determine the effect of human behaviour through radiofrequency voltage at different hertz. Brain consists of approximately ten billion neurons which are electric relay analogues. They are connected in circuit and synapses by fibres that are called axons. It is via these axons that impulses are transmitted from one neuron to another, from one group of neuron to the next. Every sensation has its code, its intensity and its duration. The pulse code of these frequencies moves along the specific neuron loops and because of that one feels all these sensations. Pulse code characteristics take place of a certain frequency that is individual. In a radiofrequency field, an oscillating current flow is propagated through one group of neuron to the next. Individual frequency with different pulse code unit can be transferred from one neuron to the next with the help of a pulse oscillator because human pulse code characteristics are individual. Impulse due to a change in momentum under electrical oscillation through neuron loops appears to an active influence to the migrating impulse with a decisive importance to human thought. In turn radio emission determines all these sensations at different hertz. To increase radiofrequency voltage at a particular hertz, human sensation turns into muscular pain as well as drowsiness to become sick. A radiofrequency of a particular hertz plays an important role of brain attack in the presence of a pulse oscillator. Indeed, sensation at different pulse code unit emerge the human characteristics in many ways such as 1) Emotion 2) Frustration 3) Passion 4) Hesitation 5) Excitement 6) Attraction 7) Sorrow 8) Confidence 9) Restless 10) Insurrection etc. Eventually, this scientific investigation reveals to an artificial human activity in a radiofrequency field.

\section{Conclusion}

Indian Television network (National Channel) in association with Star Television network (United Kingdom) continues its scientific operations through human brain around the world with reference to neurocybernetics. Neurocybernetics deals with radio emission in a vacuum to put artificial human environment around the world. Thermonuclear reaction determines X-emission in a vacuum to increase radiofrequency voltage. Representing the analysis of an artificial human environment it is stated that thermonuclear reaction prevails the destruction of humanity around the world. Civilization grows with species concentration to exhibit World War III with reference to several Windows' Channel via Earth observation satellite. A destruction of the society tends to a great loss of ethics and culture. Author himself does not know about where civilization goes. In response to author investigation it is stated that thermonuclear reaction is dangerous to civilization. 


\section{References}

[1] Ghosh, S.K. (2001) A Note on Unsteady Hydromagnetic Flow in a Rotating Channel Permeated by an Inclined Magnetic Field in the Presence of an Oscillator. Czechoslovak Journal of Physics, 51, 799-804.

https://doi.org/10.1023/A:1011622416362

[2] Ghosh, S.K. (2007) Exploitation of the Universe with Regard to Controlled Thermonuclear Fusion Reaction of the Sun. International Journal of Applied Mechanics and Engineering, 12, 571-573.

[3] Ghosh, S.K. (2007) Transmission of Radio Wave with Regard to Controlled Thermonuclear Fusion Reaction of the Sun. Advanced Studies in Theoretical Physics, 1 , 169-172.

[4] Ghosh, S.K., Beg, O.A., Zueco, J. and Prasad, V.R. (2010) Transient Hydromagnetic Flow in a Rotating Channel Permeated by an Inclined Magnetic Field with Magnetic Induction and Maxwell Displacement Current Effects. Zeitschrift fur angewandte Mathematik und Physik (ZAMP), 61, 147-169.

https://doi.org/10.1007/s00033-009-0006-2

[5] Ghosh, S.K. (2014) Dynamo Mechanism of the Sun with Reference to Magnetohydrodynamic Flows. World Journal of Mechanics, 4, 112-115.

https://doi.org/10.4236/wjm.2014.44013 